\title{
High genetic heterogeneity of Mycobacterium intracellulare isolated from respiratory specimens
}

\author{
Nicoletta Lari and Laura Rindi*
}

\begin{abstract}
Background: M. intracellulare is a frequent causative pathogen of nontuberculous mycobacteria infection that causes infections in the respiratory tract, whose incidence is increasing in many countries. This study aimed at determining the VNTR-based genetic diversity of a collection of $39 \mathrm{M}$. intracellulare human strains isolated from respiratory specimens over the last 5 years.

Results: The VNTR analysis showed that $M$. intracellulare strains displayed a high genetic diversity, indicating that the M. intracellulare genotypes are quite heterogeneous in our geographical area. Moreover, a comparison with VNTR profiles of strains from other countries confirmed that genotypes of clinical strains of $M$. intracellulare are not related to geographical origin.
\end{abstract}

Conclusions: VNTR typing has proved to be a highly discriminatory method for better understanding the molecular epidemiology of $M$. intracellulare.

Keywords: Mycobacterium intracellulare, Molecular typing, VNTR analysis

\section{Background}

Mycobacterium intracellulare, a member of Mycobacterium avium complex (MAC), is responsible for many of the human-associated nontuberculous mycobacteria pulmonary infections worldwide [1]. M. intracellulare is ubiquitous in the environment, including soil and water, and several studies reported drinking water or bathroom as sources of infection for human [2]. M. intracellulare is an important pathogen that causes infections in the respiratory tract in immunocompetent patients, showing more severe clinical characteristics and worse prognosis than infections caused by M. avium [3]. In Italy, as in many other countries worldwide, $M$. intracellulare is a frequent causative pathogen of nontuberculous mycobacteria infection and the incidence of $M$. intracellulare

*Correspondence: laura.rindi@unipi.it

Dipartimento di Ricerca Traslazionale e delle Nuove Tecnologie

in Medicina e Chirurgia, Università di Pisa, 35/39, I-56127 Pisa, Italy infections is increasing [4] . New strategies, based on the knowledge of epidemiology and biodiversity of clinical strains, aimed at the effective control of $M$. intracellulare infections in humans, are needed. The variable numbers of tandem repeats (VNTR) analysis is a rapid, highly reproducible and discriminatory genotyping method that has been successfully applied for MAC isolates, especially M. avium [5-8]. Recently, studies have been performed to select effective VNTR loci for $M$. intracellulare and to evaluate their epidemiological usefulness $[9,10]$.

In the present study, in light of the significant increase in $M$. intracellulare occurred in recent years, we determined the VNTR-based genetic diversity of a collection of M. intracellulare human strains isolated from 2015 to 2019 in order to determine molecular epidemiology and estimate the genetic relationships among $M$. intracellulare isolates in our setting. 


\section{Results and discussion}

A total of $39 M$. intracellulare human strains, isolated over a 5 year-period from the same number of TB patients resident in Tuscany, Italy, were genotyped by determining the polymorphism of a set of 16 VNTR loci [10]. Each of the 16 VNTR loci were efficiently amplified from all strains. First, the resolution provided by each VNTR locus was quantified by calculating its allelic diversity, which depends upon both the number and the distribution of the alleles, according to Selander et al. [11]. Table 1 shows the allelic diversity of the VNTR loci of our collection. Eight loci (VNTR-2, -3, -4, -5, -7, -9, -10 and -13$)$ had a high diversity index $(h \geq 0.5)$; eight loci (VNTR-1, $-6,-8,-11,-12,-14,-15$ and -16 ) achieved a medium diversity index $(0.1 \leq h \leq 0.5)$; none of the 16 loci showed a low diversity index $(\mathrm{h} \leq 0.1)$ (Fig. 1).

Table 1 VNTR allelic distribution in 39M. intracellulare clinical isolates

\begin{tabular}{|c|c|c|c|c|c|c|c|c|c|c|c|c|}
\hline \multirow[t]{2}{*}{ VNTR locus } & \multicolumn{11}{|c|}{ No. of isolates with the specified VNTR copy number } & \multirow[t]{2}{*}{ Allelic diversity } \\
\hline & 0 & 1 & 2 & 3 & 4 & 5 & 6 & 7 & 8 & 9 & 10 & \\
\hline 1 & 1 & 2 & 32 & 1 & 1 & 2 & & & & & & 0.30 \\
\hline 2 & 6 & 5 & 8 & 19 & & & & 1 & & & & 0.67 \\
\hline 3 & 15 & 14 & 10 & & & & & & & & & 0.65 \\
\hline 4 & & 17 & 4 & 10 & 7 & & & 1 & & & & 0.69 \\
\hline 5 & 11 & 4 & 24 & & & & & & & & & 0.52 \\
\hline 6 & 3 & 6 & 27 & 3 & & & & & & & & 0.47 \\
\hline 7 & 1 & 7 & 18 & 9 & & & & & 1 & & 3 & 0.69 \\
\hline 8 & 8 & 3 & 25 & 3 & & & & & & & & 0.47 \\
\hline 9 & 10 & 9 & 13 & 6 & 1 & & & & & & & 0.74 \\
\hline 10 & 1 & 13 & 5 & & 15 & 5 & & & & & & 0.70 \\
\hline 11 & & 1 & 30 & 5 & 2 & 1 & & & & & & 0.37 \\
\hline 12 & & 3 & 34 & 2 & & & & & & & & 0.21 \\
\hline 13 & 4 & & 10 & 24 & & 1 & & & & & & 0.53 \\
\hline 14 & 1 & 3 & 32 & 3 & & & & & & & & 0.30 \\
\hline 15 & & 9 & 30 & & & & & & & & & 0.34 \\
\hline 16 & 1 & 6 & 29 & 1 & 2 & & & & & & & 0.40 \\
\hline
\end{tabular}

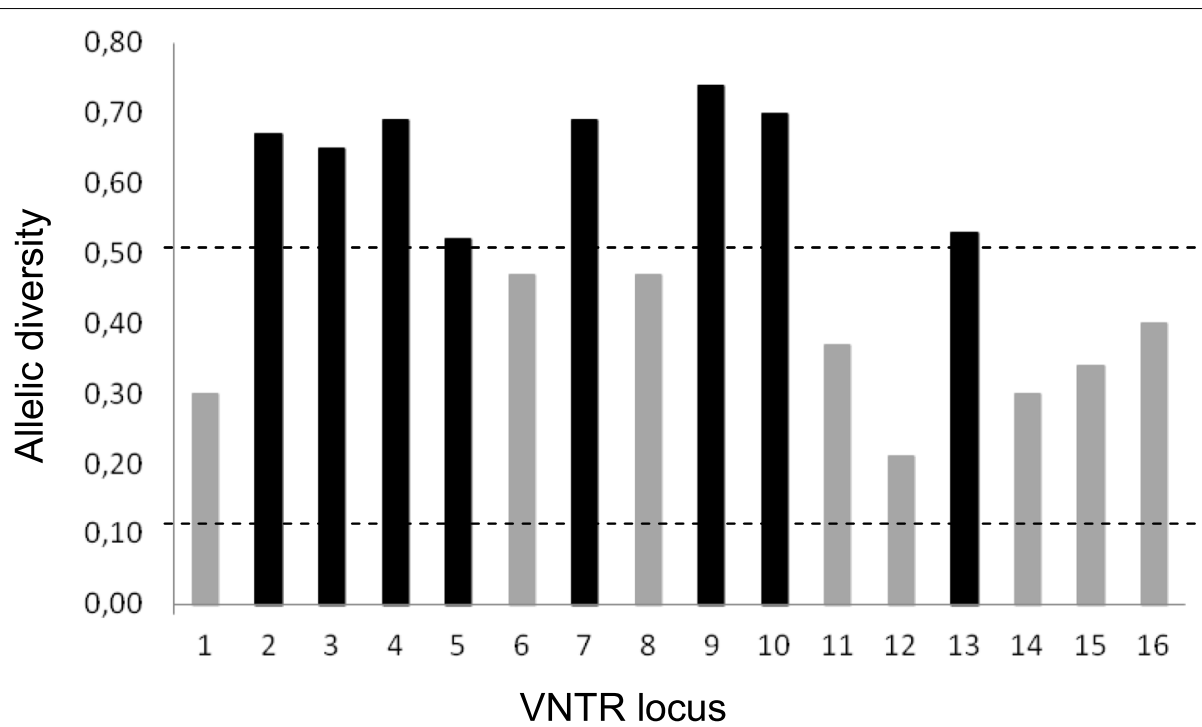

Fig. 1 VNTR allelic distribution in 39M. intracellulare clinical isolates. Black and grey bars indicate strains with high and medium diversity index, respectively 
In agreement with previous reports [10, 12-14], the discriminatory power of VNTR typing yielded an HGDI of 0.993 , confirming the great ability of the VNTR analysis to differentiate $M$. intracellulare isolates.

The VNTR analysis was used to construct a dendrogram, reported in Fig. 2, in which the VNTR patterns were ordered by similarity. Our VNTR analysis revealed 35 distinct VNTR genotypes; of these, 32 patterns were unique, while 3 patterns were shared by multiple isolates, thus yielding 2 clusters consisting of 2 strains and 1 cluster of 3 strains. The genetic relationships between the study isolates were also assessed by construction of a minimum spanning tree (Fig. 3). The minimum spanning tree, based on variations from one allele to another due to the loss or gain of one sequence at a single VNTR locus, allowed us to distinguish six clonal complexes. Complex 1 included 5 isolates, 2 of which were grouped in a cluster. The other five complexes were composed of 2 isolates with unique VNTR profile.
On the whole, the results obtained through the VNTR analysis showed that $M$. intracellulare strains displayed a high genetic diversity, indicating that the $M$. intracellulare genotypes are quite heterogeneous in our geographical area. These data differs to what has recently been demonstrated for $M$. avium subsp. hominissuis strains, isolated in the same geographical area, that displayed a close genetic relationship [15], and therefore support the hypothesis that $M$. intracellulare and $M$. avium may have different sources of infection and transmission pathways, as suggested in a recent studies $[6,14]$. However, this study has some limitations: the population size was small and phenotypic features such as drug susceptibility and clinical characteristics of the strains were not investigated.

Moreover, in order to evaluate the impact of geographical origin on $M$. intracellulare genotypes, our 16-loci VNTR results were compared with data of clinical isolates from other countries. In this regard, in addition

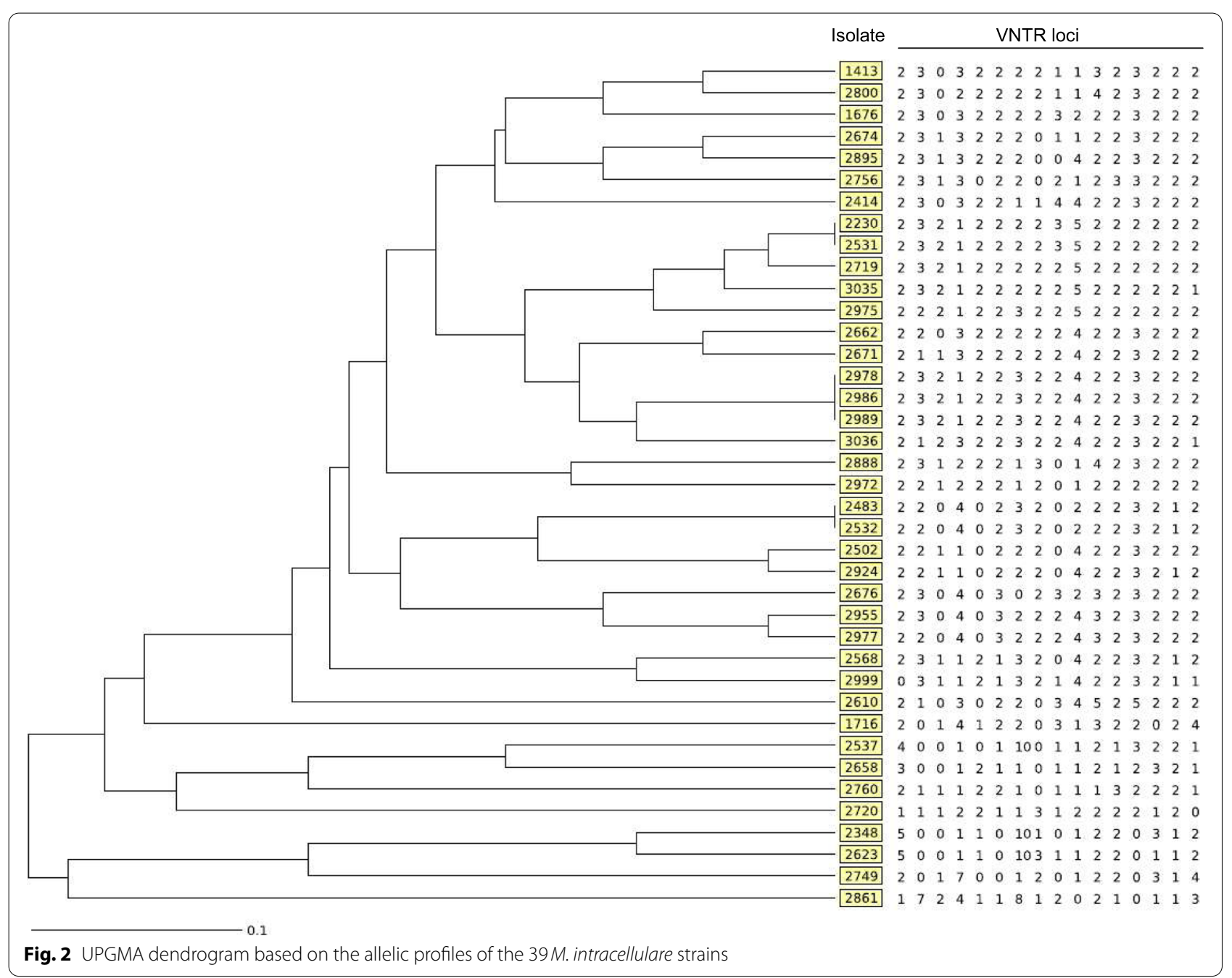




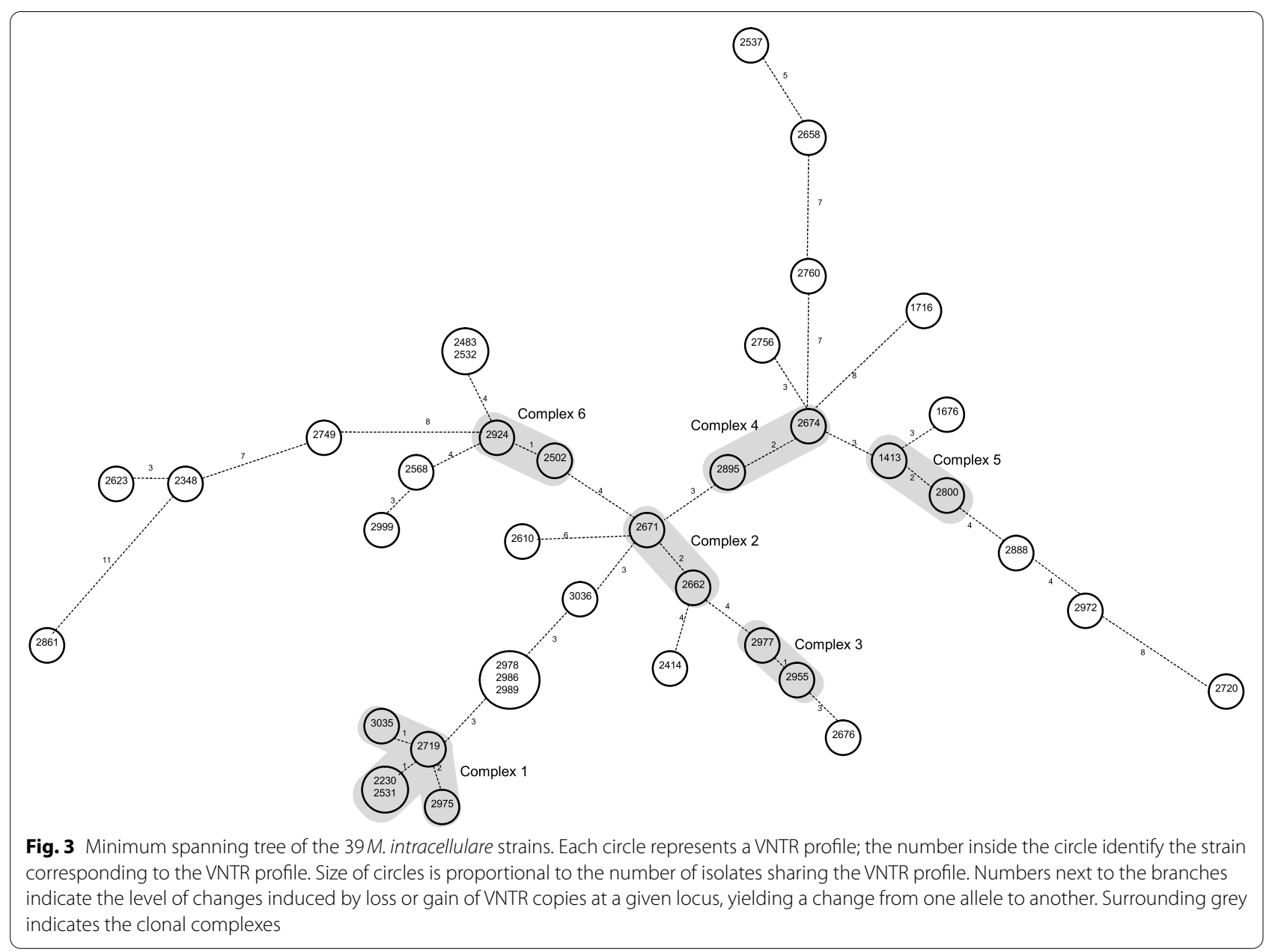

to the $39 M$. intracellulare strains characterized in this study, 226M. intracellulare clinical isolates from Japan [6, 10, 16], 115 from Korea [6, 17], 52 from China [13], 15 from United States [6] and 7 from the Netherlands [6] were considered. As shown in Fig. 4, representing the minimum spanning tree obtained by comparing a total of $454 \mathrm{M}$. intracellulare clinical strains, isolates from different countries were distributed overall, indicating that no correlation between the 16-loci VNTR genotypes and the geographical region of strains isolation was observed. This comparative analysis integrates and confirms findings of other studies demonstrating that genetic characteristics of clinical strains of $M$. intracellulare are not related to geographical origin $[6,17]$. On the other hand, the VNTR analysis, which has proved to be a very useful and highly discriminatory tool in molecular epidemiology studies, is unable to recognize species related to $M$. intracellulare, such as Mycobacterium paraintracellulare and Mycobacterium indicus pranii. The identification of clinical isolates genetically close to $M$. intracellulare, addressed by multigene sequence-based analysis [18] or comparative genomic analysis [19], is important to define virulence determinants and evolution of MAC strains causing pulmonary disease.

\section{Conclusions}

The evaluation of the genetic diversity of $M$. intracellulare strains isolated from respiratory specimens over a 5 -year period, carried out using the 16-loci VNTR analysis, provided the identification of circulating genotypes in a region of Italy. Our study showed a high genetic heterogeneity of $M$. intracellulare isolates and confirmed that genotypes of clinical strains of $M$. intracellulare are not related to geographical origin. VNTR typing has proved to be a highly discriminatory method for better understanding the molecular epidemiology of $M$. intracellulare.

\section{Methods \\ Clinical isolates}

A total of $39 \mathrm{M}$. intracellulare strains, identified by GenoType NTM-DR test (Hain Lifescience), able to discriminate between M. intracellulare and Mycobacterium 


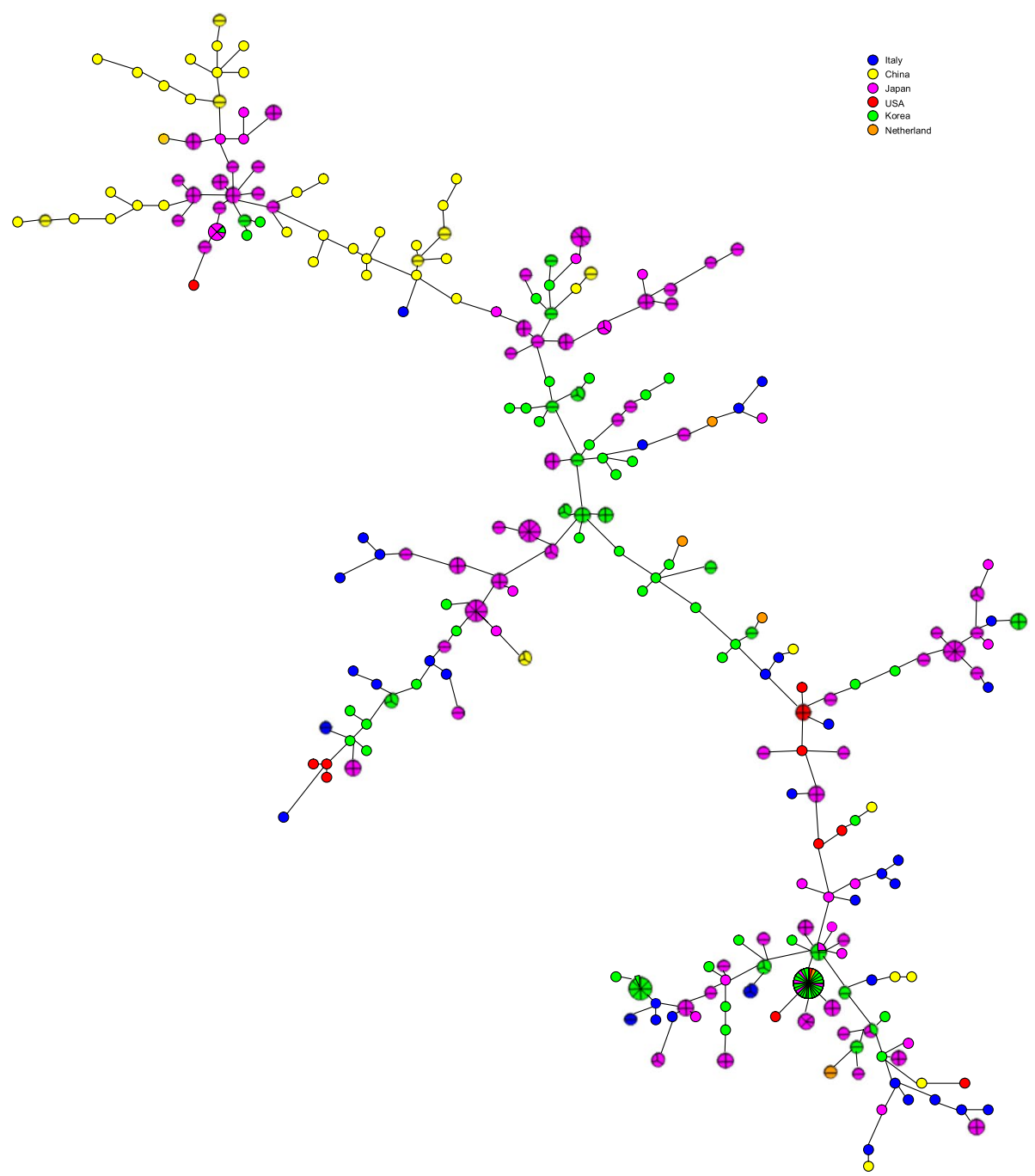

Fig. 4 Minimum spanning tree of 454 M. intracellulare strains. The genetic relationships between 39 isolates from Italy (this study), 226 from Japan $[6,10,16], 115$ from Korea [6, 17], 52 from China [13], 15 from United States [6] and 7 from The Netherlands [6] were visualized. Each circle represents a VNTR profile; size of circles is proportional to the number of isolates sharing the VNTR profile. The length of the lines connecting isolates are not proportional to the number of allelic variation between the isolates

chimaera, were isolated between 2015 and 2019 from the same number of patients (23 males and 16 females; $70 \%$ older than 65 years) resident in Tuscany, Italy, with pulmonary infections at the Laboratory of Clinical Mycobacteriology of the University Hospital of Pisa, Italy.

\section{VNTR analysis}

Genomic DNA was extracted by the cetyltrimethylammonium bromide (CTAB) method. VNTR typing was performed by PCR using specific primers for $16 \mathrm{M}$. intracellulare VNTR loci, as described previously [10]. The PCR fragments were analyzed by gel electrophoresis using 2\% NuSieve agarose (Cambrex Bio Science Rockland). For each locus, sizes of amplicons were estimated by comparison with $20 \mathrm{bp}$ and $100 \mathrm{bp}$ markers (Superladder-low; GenSura, CA, USA. VNTR profile is expressed as a string of 16 numbers, each representing the number of tandem repeats (TR) at a given VNTR position.

\section{VNTR allelic diversity and genetic relationships analysis}

The allelic diversity of the VNTR loci was calculated according to Selander's formula [11]. The global discriminatory power of complete VNTR scheme was determined using the Hunter and Gaston discriminatory index (HGDI) [20].

VNTR data were analyzed by the MIRU-VNTRplus web application available at www.miru-vntrplus.org; VNTR profile similarities were visualized by generating 
a dendrogram using the unweighted pair group method with arithmetic averages (UPGMA); the genetic relationships among the isolates were analyzed by constructing a minimum spanning tree, an undirected network in which all the VNTR profiles are linked together with the smallest possible linkages between nearest neighbours, by the UPGMA method.

\section{Acknowledgments}

Not applicable.

\section{Authors' contributions}

NL performed the experiments. LR conceived and wrote the study. All authors read and approved the final version of the manuscript.

\section{Funding}

This work was financially supported by the University of Pisa, grant "Fondi di

Ateneo, 2019".

\section{Availability of data and materials}

All data generated or analysed during this study are included in this published article.

\section{Declarations}

Ethics approval and consent to participate

Not applicable.

\section{Consent for publication}

Not applicable.

\section{Competing interests}

The authors declare that they have no competing interests.

Received: 11 October 2021 Accepted: 8 December 2021

Published online: 03 January 2022

\section{References}

1. Prevots DR, Marras TK. Epidemiology of human pulmonary infection with non-tuberculous mycobacteria: a review. Clin Chest Med. 2015;36:13-34. https://doi.org/10.1016/j.ccm.2014.10.002.

2. Nishiuchi Y, Iwamoto T, Maruyama F. Infection sources of a common nontuberculous mycobacterial pathogen, complex. Front Med. 2017;4:27. https://doi.org/10.3389/fmed.2017.00027.

3. Koh WJ, Jeong BH, Jeon K, Lee NY, Lee KS, Woo SY, et al. Clinical significance of the differentiation between Mycobacterium avium and Mycobacterium intracellulare in M avium complex lung disease. Chest. 2012;142:1482-8. https://doi.org/10.1378/chest.12-0494.

4. Rindi L, Garzelli C. Increase in non-tuberculous mycobacteria isolated from humans in Tuscany, Italy, from 2004 to 2014. BMC Infect Dis. 2016;16:44. https://doi.org/10.1186/s12879-016-1380-y.

5. Arikawa K, Ichijo T, Nakajima S, Nishiuchi Y, Yano H, Tamaru A, et al. Genetic relatedness of Mycobacterium avium subsp. hominissuis isolates from bathrooms of healthy volunteers, rivers, and soils in Japan with human clinical isolates from different geographical areas. Infect Genet Evol. 2019;74:103923. https://doi.org/10.1016/j.meegid.2019.103923.

6. Ichikawa K, van Ingen J, Koh WJ, Wagner D, Salfinger M, Inagaki T, et al. Genetic diversity of clinical Mycobacterium avium subsp. hominissuis and Mycobacterium intracellulare isolates causing pulmonary diseases recovered from different geographical regions. Infect Genet Evol. 2015;36:2505. https://doi.org/10.1016/j.meegid.2015.09.029.

7. Lande L, Alexander DC, Wallace RJ Jr, Kwait R, lakhiaeva E, Williams $M$, et al. Mycobacterium avium in community and household water, suburban Philadelphia, Pennsylvania, USA, 2010-2012. Emerg Infect Dis. 2019;25:473-81. https://doi.org/10.3201/eid2503.180336.
8. Shin Jl, Shin SJ, Shin MK. Differential genotyping of Mycobacterium avium complex and its implications in clinical and environmental epidemiology. Microorganisms. 2020;8:98. https://doi.org/10.3390/microorganisms8 010098.

9. Dauchy FA, Dégrange S, Charron A, Dupon M, Xin Y, Bébéar C, et al. Variable-number tandem-repeat markers for typing Mycobacterium intracellulare strains isolated in humans. BMC Microbiol. 2010;10:93. https:// doi.org/10.1186/1471-2180-10-93.

10. Ichikawa K, Yagi T, Inagaki T, Moriyama M, Nakagawa T, Uchiya K, et al. Molecular typing of Mycobacterium intracellulare using multilocus variable number of tandem-repeat analysis: identification of loci and analysis of clinical isolates. Microbiology. 2010;156:496-504. https://doi.org/10. 1099/mic.0.030684-0.

11. Selander RK, Caugant DA, Ochman H, Musser JM, Gilmour MN, Whittam TS. Methods of multilocus enzyme electrophoresis for bacterial population genetics and systematics. Appl Environ Microbiol. 1986;51:873-84. https://doi.org/10.1128/aem.51.5.873-884.1986.

12. Kim SY, Lee ST, Jeong BH, Park HY, Jeon K, Kim JW, et al. Genotyping of Mycobacterium intracellulare isolates and clinical characteristics of lung disease. Int J Tuberc Lung Dis. 2013;17:669-75. https://doi.org/10.5588/ ijtld.12.0575.

13. Zhao $X$, Wang $Y$, Pang $Y$. Antimicrobial susceptibility and molecular characterization of Mycobacterium intracellulare in China. Infect Genet Evol. 2014;27:332-8. https://doi.org/10.1016/j.meegid.2014.07.032.

14. Zheng HW, Pang Y, He GX, Song YY, Zhao YL. Comparing the Genotype and Drug Susceptibilities between Mycobacterium avium and Mycobacterium intracellulare in China. Biomed Environ Sci. 2017;30:517-25. https:// pubmed.ncbi.nlm.nih.gov/28756811/. https://doi.org/10.3967/bes2017. 068.

15. Genua F, Menichini M, Lari N, Rindi L. Genotyping and clarithromycin susceptibility testing of Mycobacterium avium subsp. hominissuis isolated in Tuscany, Italy. Infect Genet Evol. 2018:65:144-9. https://doi.org/10.1016/j. meegid.2018.07.032.

16. Fujita K, Ito Y, Hirai T, Kubo T, Maekawa K, Togashi K, et al. Association between polyclonal and mixed mycobacterial Mycobacterium avium complex infection and environmental exposure. Ann Am Thorac Soc. 2014;11:45-53. https://doi.org/10.1513/annalsats.201309-297oc.

17. Shin Jl, Ha JH, Lee DH, Choi JG, Kim KM, Lee SJ, et al. Comparative evaluation of band-based genotyping methods for Mycobacterium intracellulare and its application for epidemiological analysis. Microorganisms. 2020;8:1315. https://doi.org/10.3390/microorganisms8091315.

18. Kim SY, Park HY, Jeong BH, Jeon K, Huh HJ, Ki CS, et al. Molecular analysis of clinical isolates previously diagnosed as Mycobacterium intracellulare reveals incidental findings of "Mycobacterium indicus pranii" genotypes in human lung infection. BMC Infect Dis. 2015;15:406. https://doi.org/10. 1186/s12879-015-1140-4.

19. Tateishi Y, Ozeki Y, Nishiyama A, Miki M, Maekura R, Fukushima Y, et al. Comparative genomic analysis of Mycobacterium intracellulare: implications for clinical taxonomic classification in pulmonary Mycobacterium avium-intracellulare complex disease. BMC Microbiol. 2021;21:103. https:// doi.org/10.1186/s12866-021-02163-9.

20. Hunter PR, Gaston MA. Numerical index of the discriminatory ability of typing systems: an application of Simpson's index of diversity. J Clin Microbiol. 1988;26:2465-6. https://doi.org/10.1128/jcm.26.11.2465-2466. 1988.

\section{Publisher's Note}

Springer Nature remains neutral with regard to jurisdictional claims in published maps and institutional affiliations. 